\title{
Use of Operational Microwave Link Measurements for the Tomographic Reconstruction of 2-D Maps of Accumulated Rainfall
}

\author{
Michele D'Amico, Senior Member, IEEE, Andrea Manzoni, and Gian Leonardo Solazzi
}

\begin{abstract}
In this letter, we show how an operational microwave link network can be used to reconstruct 2-D fields of accumulated rainfall over a limited area at ground level. To this purpose, we use a tomographic technique that exploits the relation between rainfall rate and the attenuation experienced by the microwave links of the network. The links located in Italy operate at K-band and are managed by an Internet service provider. The results are validated by comparing the reconstructed rainfall maps with those derived from a network of conventional rain gauges. Finally, we assess by simulation the impact of the network's density and topology on the accuracy of the reconstruction algorithm.
\end{abstract}

Index Terms-Hydrology, measurement campaign, microwave links, rain field, tomography.

\section{INTRODUCTION}

$\mathbf{R}$ ELIABLE measurement of rainfall is crucial hydrological models, whether they are used for real-time floods prevention or water resources planning. Generally, rainfall measurements are collected by dedicated instruments like radars and rain gauges, which lack, respectively, in terms of quantitative reliability and time resolution. To overcome these restrictions, in the recent past, a new method for estimating rainfall has been proposed: it exploits the attenuation that rain induces on ground-based microwave links; a comprehensive review can be found in [1].

Promising experimental results were presented in the last decade: D'Amico et al. [2] derived the path-averaged rainfall rate from point-to-point link measurements, while Overeem et al. [3], [4] derived the rain field over the Netherlands interpolating attenuation data gathered on a country-wide scale network.

These works confirmed the possibility to estimate rain rate from attenuation, but none of them applied a tomographic technique to the measured data. A tomographic technique, like the ones first proposed by Giuli et al. [5]-[7], is possibly the best way for an accurate reconstruction of the rainfall

Manuscript received April 21, 2016; revised June 30, 2016 and August 30, 2016; accepted September 25, 2016. Date of publication October 18, 2016; date of current version December 7, 2016.

M. D'Amico and A. Manzoni are with the Dipartimento di Elettronica, Informazione e Bioingegneria, Politecnico di Milano, 20133 Milan, Italy (e-mail: michele.damico@polimi.it; andrea.manzoni@polimi.it).

G. L. Solazzi is with the Radio Network Planning, NGI S.p.A, 21052 Busto Arsizio, Italy (e-mail: gianleonardo.solazzi@gmail.com). field, starting from attenuation data. While tomography has been widely investigated through simulation, it has never been applied to measurements collected by operational microwave links [8]-[10].

In this letter, we apply a tomographic technique to attenuation measurements gathered by an operational microwave network located in Italy; the objective is to reconstruct 2-D fields of accumulated rainfall. This letter is organized as follows. We describe the experimental setup first, and then we briefly illustrate how to apply the tomographic technique. Finally, we validate the procedure by comparing the reconstructed field with that derived from colocated rain gauges.

\section{Microwave Link and Rain Gauge Networks}

The microwave network consists of three bidirectional links (on average $6 \mathrm{~km}$ long), operating at $25 \mathrm{GHz}$ with vertical polarization, located in the Treviso district, Northern Italy. The received power level is sampled at $8 \mathrm{~Hz}$ and quantized with a resolution of $3 \mathrm{~b} / \mathrm{dB}$; eight samples are then averaged to obtain one sample/s, so as to filter out fast scintillations. The receiver dynamic range is about $60 \mathrm{~dB}$ (i.e., from about -90 to $-30 \mathrm{dBm}$ ); no attempts were made to calibrate the receivers' Automatic Gain Control (AGC) response.

The network is operational and it is used by NGI (an Internet Service Provider (ISP) company) to interconnect the company's access points. We performed a one-month measurement campaign in September 2015, storing 86400 attenuation samples per link per day.

For the ground "truth," we relied on a network of nine rain gauges, managed by the Environmental Protection Agency of Veneto [11], which almost uniformly covers a square region (32 km in side) that surrounds the links (Fig. 1). Regrettably, the rain gauge data available for this experiment were limited to daily rainfall accumulations.

Before implementing the tomographic reconstruction technique, we verified that the daily rain accumulations derived from the two sets of measurements (i.e., link attenuations and rain gauges) were consistent. The daily accumulations "measured" by the link can be computed by summing up the 864001 1-s path-averaged accumulations $\bar{A}_{\text {sec }}$, expressed in millimeters

$$
\bar{A}_{\mathrm{sec}}=1 / 3600 \cdot\left[p_{\text {link }} /(k L)\right]^{\frac{1}{\alpha}}
$$

where $p_{\text {link }}$ is the attenuation in decibels measured on the link at a given second, $L$ is the length of the link in kilometers, 


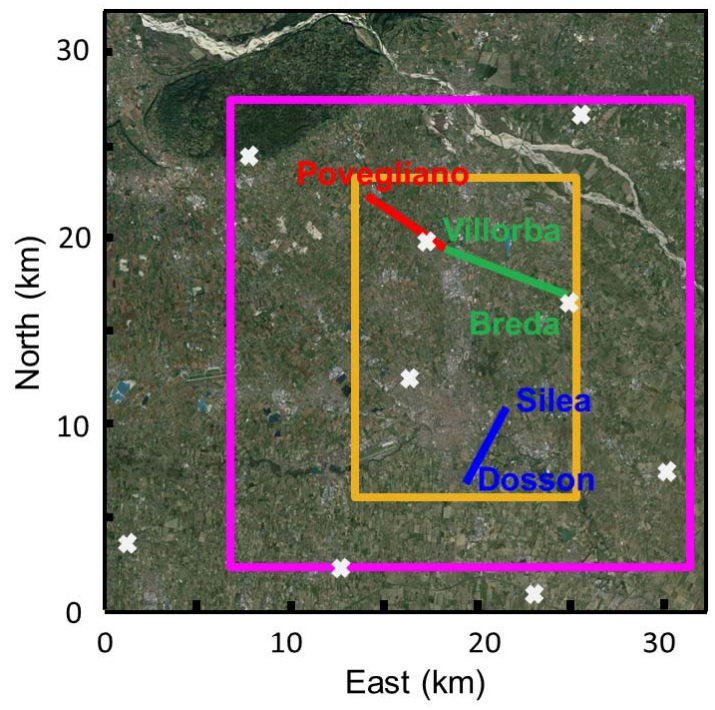

Fig. 1. Geographic region of interest, equipped with three links (colored lines) and nine rain gauges (white crosses). The monitored area is in magenta and the link area is in yellow.

and $k$ and $\alpha$ are coefficients taken from ITU-R P.838 recommendation [12], which mainly depend on the frequency and polarization of the link. For the sake of brevity, we show only the comparison referred to one link (Fig. 2), which benefits from the closeness of two rain gauges.

The use of fixed parameters in (1) inevitably introduces approximations in the estimation of the rainfall accumulation, since $k$ and $\alpha$ depend on drop size distribution, drop shape, and temperature. Another potential source of error is rainfield inhomogeneities along the measurement path; in fact, the estimated averages may be biased due to the nonlinear relation between $A$ and $R$, the impact being strongly dependent on the frequency of operation. For our frequency $(25 \mathrm{GHz})$, the bias is negligible, since the parameter $k$ is very close to unity [13]. Another possible source of error is the nonideal behavior of the communication equipment, in particular as regards system calibration and linearity. For this experimental campaign, no attempts were made to calibrate the equipment or to characterize the response of the AGC as a function of ambient temperature, power level, and so on. The idea, in fact, was to use the available microwave network "as is."

To correctly estimate the attenuation $p_{\text {link }}$ needed in (1), we have to determine the "baseline" signal level, defined as the average signal level in the last supposedly "dry" interval preceding the rain event. To this purpose, we first filter the signal-level time series to remove scintillations, artifacts, and spikes (which may be caused by devices malfunction, noise, and clear-air propagation effects like multipath). We then label as "wet" the time intervals where the signal level is attenuated in excess of $2.5 \mathrm{~dB}$ with respect to the maximum signal level measured during the previous $24 \mathrm{~h}$ (this threshold value was optimized by comparing gauge readings and measured attenuation throughout September 2015). Once wet/dry periods are identified and the baseline level is calculated, attenuation due to rain is obtained by subtraction; we are aware that there may be contributions due to water vapour variations, but those cannot be removed without ancillary instrumentation. It is worth noting that this method for the classification of wet/dry periods relies only on the power received individually by each single link; in fact, since the available links are separated by distances that can be larger than $10 \mathrm{~km}$, the spatial decorrelation of rain prevents us from using techniques that jointly consider more than one link [14].

\section{TOMOGRAPHIC TECHNIQUE}

After this preliminary validation, we used the attenuations measured simultaneously by the three links to reconstruct the rainfall field over the $25 \times 25 \mathrm{~km}^{2}$ "monitored" area shown in Fig. 1 (inside the magenta box). The tomographic technique that we implemented is the one presented by Giuli et al. [5]. We want to point out that our objective in this letter is neither to propose a new tomographic method nor to improve an existing one: we want to assess if an operational microwave network can be used to estimate the accumulated rainfall over an area through tomography, confirming or disproving what has been so far inferred through simulations.

The adopted technique calculates, for each time step, the spatial distribution of specific attenuation $\hat{\gamma}(x, y)$ starting from the total attenuation measured along the links. To this purpose, we use a set of $N$ basis functions $b_{n}(x, y)$, one for each square subregion in which we partition the monitored area

$$
\hat{\gamma}(x, y)=\sum_{n=1}^{N} \gamma_{n} \cdot b_{n}(x, y)
$$

where $x$ and $y$ are ranging from 0 to $25 \mathrm{~km}$. The basis functions in (2) are selected so that they are first-order derivable polynomials that provide local continuity to the reconstructed function $\hat{\gamma}(x, y)$. The mathematical expression of the $n$th basis function is given by

$$
b_{n}(x, y)=\Lambda\left(\frac{x-r_{n}}{\Delta}\right) \cdot \Lambda\left(\frac{y-s_{n}}{\Delta}\right)
$$

with $\Lambda(u)$ being the roof-function described by [15] and $\Delta \times \Delta$ and $\left(r_{n}, s_{n}\right)$ the subregion size and center, respectively. In order to solve (2), we need to calculate, for each subregion, the expansion coefficients $\gamma_{n}$, which allow finding the solution of the following ill-conditioned linear system:

$$
p=A \cdot \gamma+e
$$

where $p$ is a vector of the attenuations measured over the $M$ links, $e$ is the error vector between the estimated and measured attenuations, and $A$ is an $M \times N$ matrix whose generic element $a_{m, n}$ is calculated by integrating the $n$th basis function along the $m$ th link path. A solution for (4) can be found by applying the following recursive algorithm:

$$
\gamma_{l+1}=\gamma_{l}+1 / k\left[-\left(I+b_{0} B\right) \gamma_{l}+a_{0} A^{T}\left(p-A \gamma_{l}\right)\right]
$$

where $k$ is a constant equal to the average between the minimum and the maximum eigenvalues of the matrix $(I+$ $\left.b_{0} B+a_{0} A^{T} A\right)$.

All tomographic reconstructions reported in this letter have been performed assuming $a_{0}$ and $b_{0}$ in (5) to be constant 


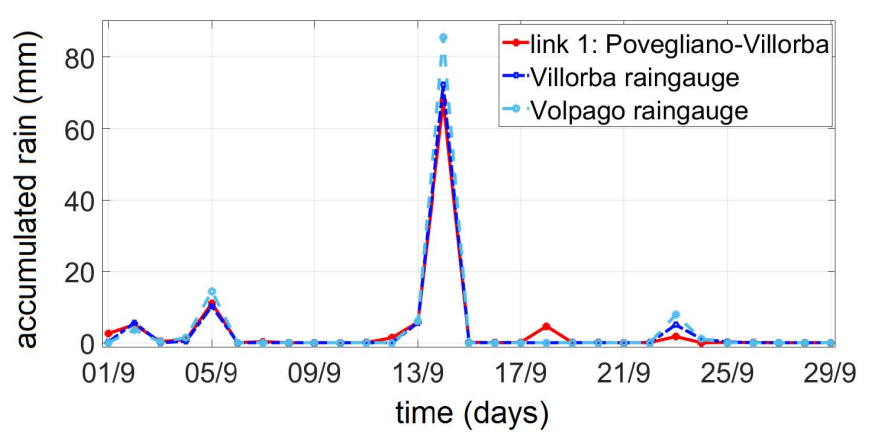

Fig. 2. Daily rain accumulation (September 2015), as measured by two rain gauges (blue dashed lines) and a microwave link (red solid line).

in time. The values of $a_{0}$ and $b_{0}$ have been determined by applying the tomographic technique and comparing the predictions with the observations carried out in two rainy days (September 5, 2015 and September 23, 2015), selected as "calibration days." Following the strategy described in [5], we found that $a_{0}=150$ and $b_{0}=70$ minimize the relative error of the path-averaged rainfall daily accumulation to values lower than $2 \%$.

Once the $a_{0}$ and $b_{0}$ coefficients have been determined, it is possible to apply the tomographic technique to the event(s) of interest; since we want to reconstruct the daily accumulation, we iterate the procedure for all seconds of the selected day. The results presented here are relative to September 14, 2015, the day of the month with the largest precipitation [more than $70 \mathrm{~mm}$ (see Fig. 2)]. We applied the technique over the monitored area $(25 \mathrm{~km} \times 25 \mathrm{~km})$, divided into 64 subregions, obtaining the 2-D rain field shown in Fig. 3(a); all the images presented are relative to the smaller "link" area $(12 \mathrm{~km} \times 17 \mathrm{~km}$, the yellow box in Fig. 1). The monitored area is larger than the link area because when we move away from the links, the uncertainty in the rainfall estimates greatly increases, due to the lack of input data which condition the system (4) to be solved. Moreover, the solution in the subregions neighboring with the boundary of the monitored area may be invalid due to numerical edge effects and must be discarded.

Although the number of available links is limited, the estimation error experienced along the links is remarkably low: by integrating for each second the function $\hat{\gamma}(x, y)$ along the links and converting specific attenuation into rainfall rate, we obtained reconstructed path-averaged accumulations that differ for less than $1 \mathrm{~mm}$ from the attenuation-imposed accumulations. Regrettably, the limited number of links available for this study does not allow us to perform a meaningful comparison of the different tomographic techniques proposed throughout the year [5]-[7], [16].

\section{ReCONSTRUCTED RAin Fields: Attenuation Versus Gauges}

The quality of the reconstructed field shown in Fig. 3(a) can be assessed by comparing it with that of an accumulation map, which is, in turn, obtained from rain gauge measurements
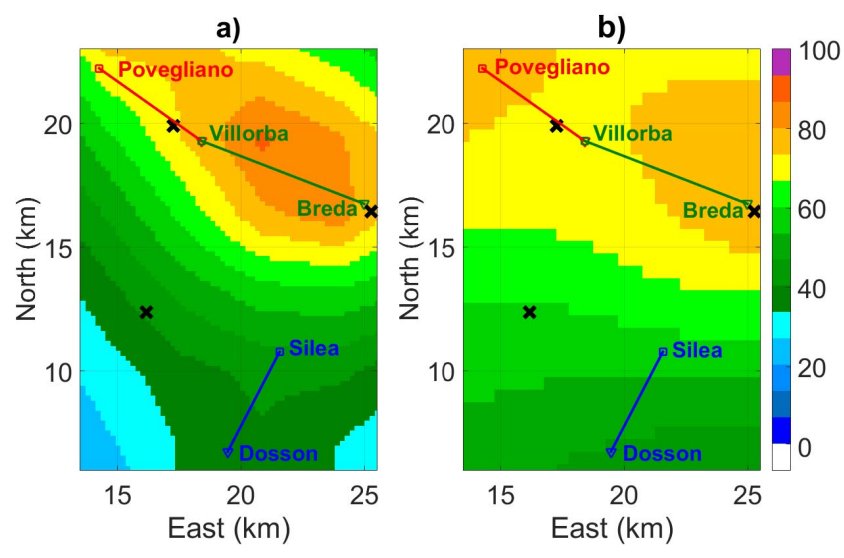

Fig. 3. Daily rain accumulation ( $\mathrm{mm})$ in the link area for 14 September (a) reconstructed with tomography from measured link attenuation and (b) measured by rain gauge network.
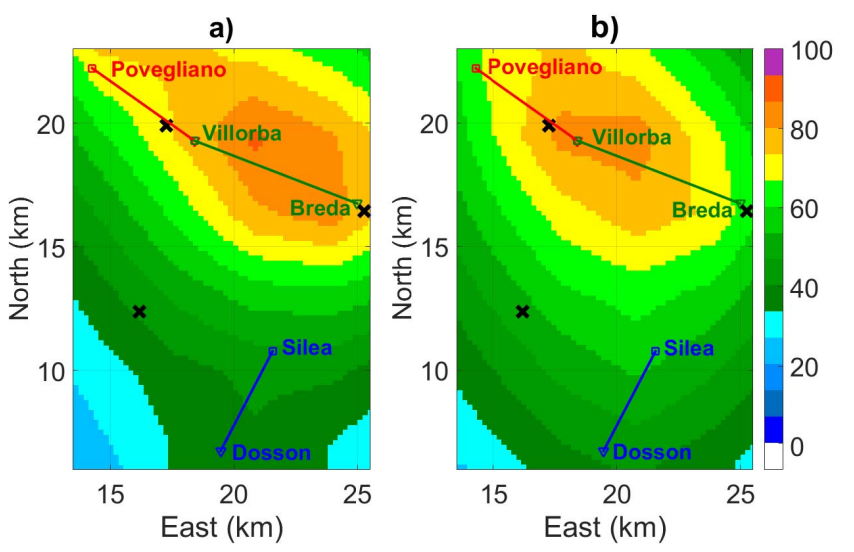

Fig. 4. Daily rain accumulation ( $\mathrm{mm}$ ) for 14 September reconstructed with tomography from (a) measured attenuations and (b) synthetic attenuations.

by applying a kriging interpolation [4], [17] (tomography, in fact, cannot be applied to point measurements). Regrettably, only daily rainfall accumulations were available. From the acquisitions of the nine rain gauges shown in Fig. 1, we reconstructed the map shown in Fig. 3(b), which can be compared with that obtained from the tomography.

Despite the small number of links, the short length of the links, the nonoptimized position of the links over the region, and the random position of rain gauges relatively to the links, Fig. 3 shows important similarities between the two maps: an increasing trend is evident, going from South to North, with accumulations up to $80-85 \mathrm{~mm}$ on both maps near the second link. On the other hand, moving away from the area directly covered by the links, the reconstructed values in Fig. 3(a) gradually lose their physical meaning.

We verified that the discrepancy between the two maps is strongly correlated $(\rho=0.91)$ with the distance between the considered point and the nearest link, with the performance worsening almost linearly as the distance increases, with a rate of about $2.5 \mathrm{~mm} / \mathrm{km}$. Moreover, as expected, the accuracy of the tomographic reconstruction is higher where we can rely on the measurements from two links. Overall, the discrepancy 

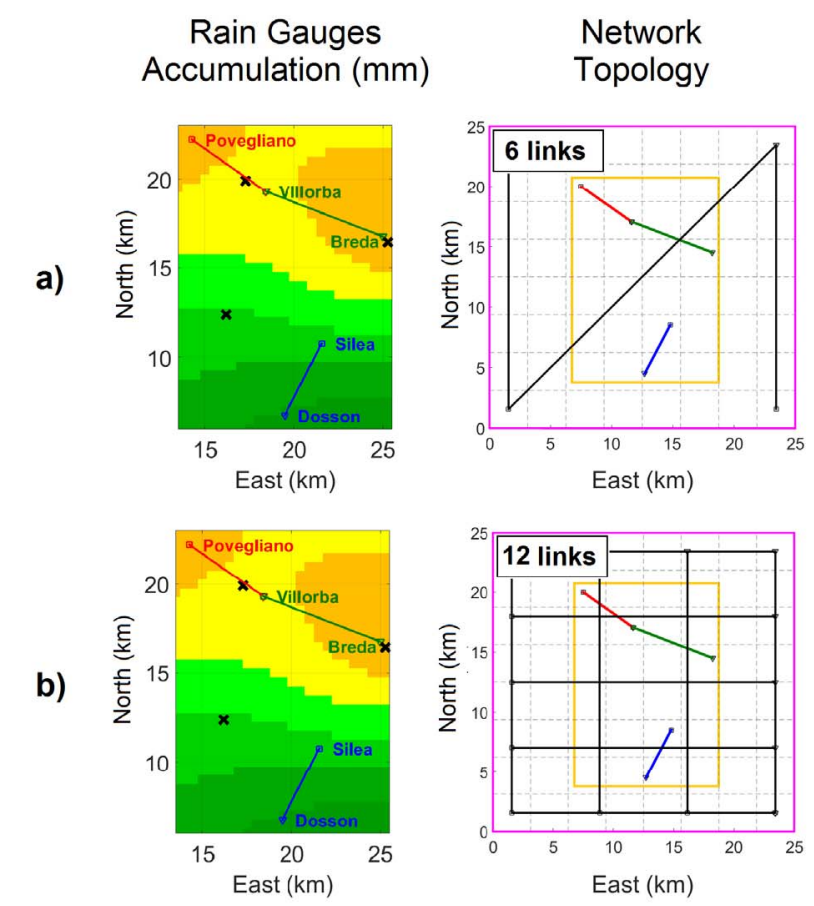

b)
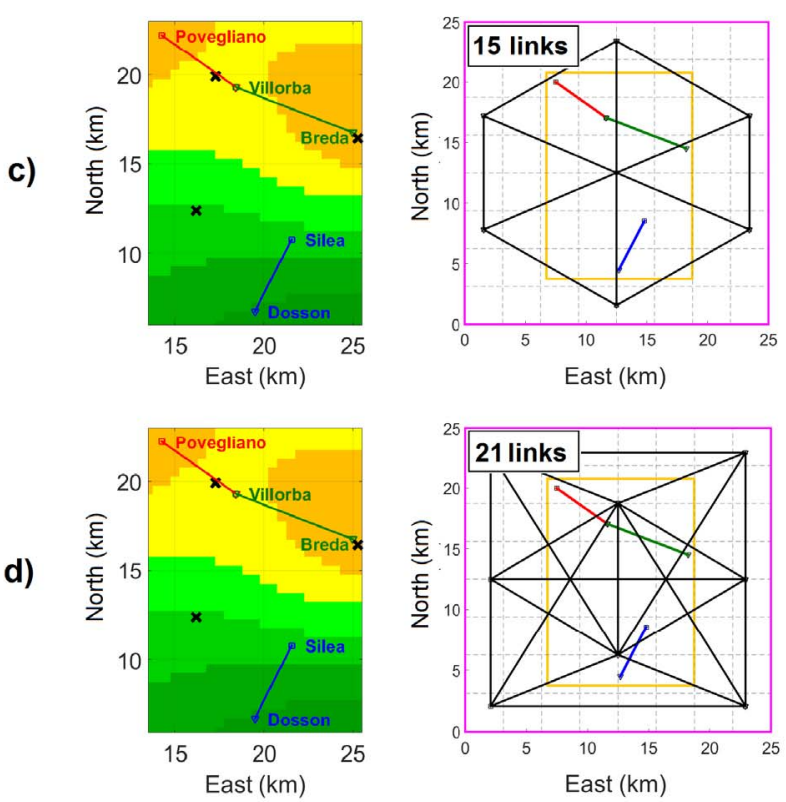

Tomography Reconstruction (mm)
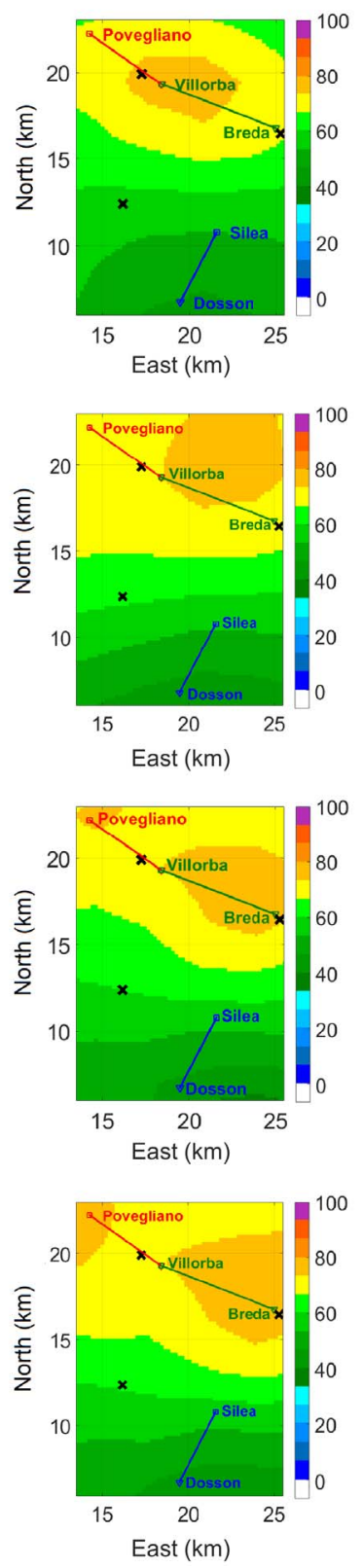

Fig. 5. Network topology simulations. (a) Network with 6 links. (b) Network with 12 links. (c) Network with 15 links. (d) Network with 21 links. (Left) Gauge accumulation from which we derived synthetic attenuations. (Middle) Network topology simulated. (Right) Result of the tomographic reconstruction.

between Fig. 3(a) and (b) does not exceed, on average, $10 \mathrm{~mm}$ (corresponding to an error of about 15\%) for all the points whose distance from the nearest link is smaller than $2.5 \mathrm{~km}$.

When comparing the two maps, we must take into account the fact that tomographic reconstruction carried out with only three links may have intrinsic limitations. To prove that the observed discrepancy is mainly due to the limited number of links and not to the tomographic technique itself, we performed the following simulation: we located "virtual" microwave links on the rain gauge-derived precipitation maps, in the same locations of the actual links; we then calculated synthetic attenuation values by converting precipitation into attenuation through the Recommendation ITU-R P.838 [12]. In the simulations, we tried to comply with the technical specifications of the actual links as much as possible (e.g., quantization of the signal level); however, we did not add noise or other artifacts since we already have significant uncertainties, due to the lack of knowledge of the drop size distribution and so on. On these synthetic values, we applied the tomographic algorithm. The comparison between the two tomographic reconstructions, which we call for brevity measured and synthetic tomographies, is reported in Fig. 4. The level of agreement between the two images is significant and it allows us to affirm that the tomographic technique applied 
to measured attenuation is reliable and that the discrepancy between reconstructed and rain gauge-derived precipitation maps may be due to-in our case - the low density of links; this point will be discussed in the next section.

\section{Synthetic Attenuations From RAIN GAUGE MEASUREMENTS}

As a next step, we investigated via simulation the impact of links' density and network topology on the quality of the tomographic reconstruction; without having the ambition to draw general conclusions, the objective is to acquire some sensitivity on the (approximate) number/density of links needed to achieve a given accuracy for our scenario.

To this purpose, we assumed the rainfall field shown in Fig. 3(b) as the "ground truth" (for this exercise, it needs only to be realistic, not real), we located "virtual" links on the map (black lines in Fig. 5) and computed synthetic attenuations by converting precipitation into attenuation through [12], and then we applied the tomographic reconstruction technique to the synthetic attenuations experienced by the virtual links.

The different topologies that we simulated were designed trying to cover as uniformly as possible the monitored area; we performed seven simulations, from a minimum of 3 links to a maximum of 21 links. Fig. 5 shows the daily rain accumulation reconstructed using $6,12,15$, or 21 virtual links; with 21 links [Fig. 5(d)], the agreement with the map in Fig. 3(b) is remarkable; quantitatively, with 21 links uniformly distributed over a region of $625 \mathrm{~km}^{2}$, it is possible to estimate the rain field with a discrepancy lower than $2 \mathrm{~mm}$ over the whole area.

\section{CONCLUSION}

In this letter, we applied a microwave tomographic technique for the reconstruction of 2-D fields of rainfall accumulation, exploiting the attenuation measured by three operational communication links located in Italy. The main advantage of the tomographic technique is that it is possible to reconstruct the accumulated rain field over the entire monitored area using a reasonable number of operational communication links (for which the attenuation measurement is a by-product), without installing any additional ad hoc infrastructure and significantly improving the estimation, which can be obtained through simple interpolation. Despite the very limited number of links, the reconstructed rain map compared satisfactorily with that derived by a colocated rain gauge network. We have also shown by simulation that by increasing the network's density, the accuracy of the reconstruction algorithm improves and that the observed discrepancies are mainly due to the limited number of links available in this context.

\section{REFERENCES}

[1] H. Messer and O. Sendik, "A new approach to precipitation monitoring: A critical survey of existing technologies and challenges," IEEE Signal Process. Mag., vol. 32, no. 3, pp. 110-122, May 2015.

[2] M. D’Amico, G. Mannucci, and M. Pinotti, "Microwave links used as electromagnetic raingauges: A real time pilot experiment in Italy," in Proc. Eur. Conf. Antennas Propag., Nice, France, Nov. 2006, pp. 1-4.

[3] A. Overeem, H. Leijnse, and R. Uijlenhoet, "Country-wide rainfall maps from cellular communication networks," Proc. Nat. Acad. Sci. USA, vol. 110, no. 8, pp. 2741-2745, 2013.

[4] A. Overeem, H. Leijnse, and R. Uijlenhoet, "Retrieval algorithm for rainfall mapping from microwave links in a cellular communication network," Atmos. Meas. Tech., vol. 9, no. 5, pp. 2425-2444, 2016.

[5] D. Giuli, A. Toccafondi, G. B. Gentili, and A. Freni, "Tomographic reconstruction of rainfall fields through microwave attenuation measurements," J. Appl. Meteorol., vol. 30, pp. 1323-1340, Feb. 1991.

[6] D. Giuli, L. Facheris, and S. Tanelli, "A new microwave tomography approach for rainfall monitoring over limited areas," Phys. Chem. Earth, vol. 22, nos. 3-4, pp. 265-273, 1997.

[7] D. Giuli, L. Facheris, and S. Tanelli, "Microwave tomographic inversion technique based on stochastic approach for rainfall fields monitoring," IEEE Trans. Geosci. Remote Sens., vol. 37, no. 5, pp. 2536-2555, Sep. 1999.

[8] F. Cuccoli, L. Facheris, and S. Gori, "Radio base network and tomographic processing for real time estimation of the rainfall rate fields," in Proc. IEEE IGARSS, Cape Town, South Africa, Jul. 2009, pp. 121-124.

[9] F. Cuccoli and L. Facheris, "Rainfall monitoring based on microwave link attenuation and related tomographic processing," in Proc. Adv. Radar Remote Sens. Tyrrhenian Workshop, Naples, Italy, Sep. 2012, pp. $172-176$.

[10] A. Zinevich, P. Alpert, and H. Messer, "Estimation of rainfall fields using commercial microwave communication networks of variable density," Adv. Water Resour, vol. 31, no. 11, pp. 1470-1480, Nov. 2008.

[11] Environmental Protection Agency of Veneto Weather Data Archive, accessed on Feb. 2016. [Online]. Available: http://www.arpa.veneto.it/ bollettini/storico/

[12] Specific Attenuation Model for Rain for Use in Prediction Methods, document ITU-R Rec. P.838-3, 2005. [Online]. Available: http://www.itu.int/rec/R-REC-P.838

[13] D. Atlas and C. W. Ulbrich, "Path- and area-integrated rainfall measurement by microwave attenuation in the 1-3 cm band," J. Appl. Meteorol., vol. 16, pp. 1322-1331, Sep. 1977.

[14] A. Overeem, H. Leijnse, and R. Uijlenhoet, "Measuring urban rainfall using microwave links from commercial cellular communication networks," Water Resour. Res., vol. 47, no. 12, pp. 1-16, Dec. 2011.

[15] G. T. Herman and A. Lent, "Iterative reconstruction algorithms," Comput. Boil. Med., vol. 6, no. 4, pp. 273-294, Oct. 1976.

[16] O. Sendik and H. Messer, "On the reconstructability of images sampled by random line projections," in Proc. IEEEI, Eilat, Israel, Nov. 2012, pp. 1-5.

[17] N. Cressie, "The Origins of Kriging," Math. Geol., vol. 22, no. 3, pp. 239-252, 1999. 